\title{
Real-Time Tiger Detection using YOLOv3
}

\author{
Md. Nazmus Sakib Ohee \\ Department of CSE \\ Sylhet International University Shamimabad, \\ Bagbari, Sylhet 3100, Bangladesh
}

\author{
M. A. G. Asif \\ Department of CSE, \\ Sylhet International University Shamimabad, \\ Bagbari, Sylhet 3100, Bangladesh
}

\begin{abstract}
Based on the current population of tigers around the world and high tiger killing rate in forest adjacent area, there is a major need of automated visual surveillance to safeguard the tourists and civilians in forest adjacent area as well as decrease tiger killing rate due to the presence of tigers in residential areas as predators. The objective of this paper is to detect tigers in real-time, visually. The proposed method is using YOLOv3 algorithm and comparing the success rate with the template matching approach. A dataset of 1644 tiger images was collected with all possible angles and applied to train the YOLOv3 model. Then test dataset of 10 images was used to validate the results of YOLOv3. The detector performed exceptionally well to detect tiger in different images with different rotations, providing $80 \%$ accuracy. A real time environment can be ideal for using it at the full capacity.
\end{abstract}

\section{Keywords}

Tiger detection; YOLOv3; feature extraction; object detection; Supervisely.

\section{INTRODUCTION}

Humans spur the main factors behind the endangerment of tigers due to the increasing number of human populations clashing and tampering with the original boundaries of forest [1]. A total of 13 tigers have been killed by people in one and a half decades in Bangladesh. During the period, around 265 people were killed in tiger attacks [2] in different areas close to the Sundarban mangrove region, according to government statistics of Bangladesh.

The solution of the problem is monitoring and early detection using control camera and surveillance system which can help to alert officials when tigers approach localities and save them from falling prey to the humans. In order to implement the above solution, this paper presents a real-time tiger detection method based on YOLOv3 (You Only Look Once) [3] deep learning network trained with 1644 images containing 1757 tiger objects annotated using Supervisely [4] and compares the output result with the results of [5].

The paper is organized as follows; section 2 describes related work, 3 Methodology, 4 Results and 5 Conclusion.

\section{RELATED WORK}

The advancement of computer vision is now encouraging researchers to find out solutions for real-time problems with the help of machine learning algorithms.

The research work in [5], researchers implemented a tiger identification system using template matching approach based on edge patterns and achieved an accuracy rate of $50 \%$.

In other work [6] researchers attempted to reduce tiger-human conflict in and around the Sundarbans, Bangladesh, from
August 2005 to January 2007 using the domestic dog. The system was not automated.

For solving some computer vision related problems researchers implemented YOLOv3 in Rail Surface Defect Detection [7], Field Wheat Ears Count [8], Gun Detection System [9]. These illustrate the implementation possibility of YOLOv3 for tiger detection.

\section{METHODOLOGY}

\subsection{Dataset}

A dataset of 1644 images was collected containing tigers with different position and orientation. YOLOv3 has been trained and validated using Supervisely image annotation and cluster to evaluate the real-time tiger detection system. Figure-1 shows some of the examples from the dataset used for training the model.
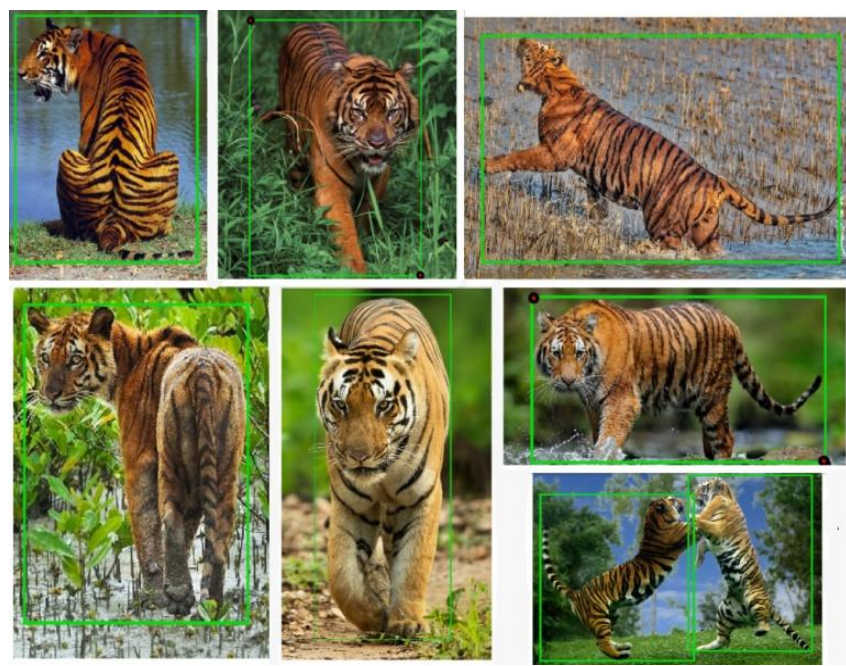

Figure-1: Images used for training YOLOv3 model

\subsection{YOLOV3 Algorithm}

YOLOv3 is a target detection model proposed by Redmon J. It transforms the detection problem into a regression problem. Adopts the end to end idea, completely passes the target detection task to the deep learning network. The algorithm uses daknet-53 network as backbone to achieve multi-scale prediction. When training the input images are divided into $\mathrm{S} * \mathrm{~S}$ grids. Each grid cell predicts $\mathrm{N}$ bounding boxes.

Yolov3 predicts bounding boxes using dimension clusters as anchor boxes and predicts 4 coordinates for each bounding box. It predicts an object score for each bounding box using logistic regression.

Figure-2 shows the image detection principle and Figure-3 shows the block diagram of YOLOv3 algorithm. 


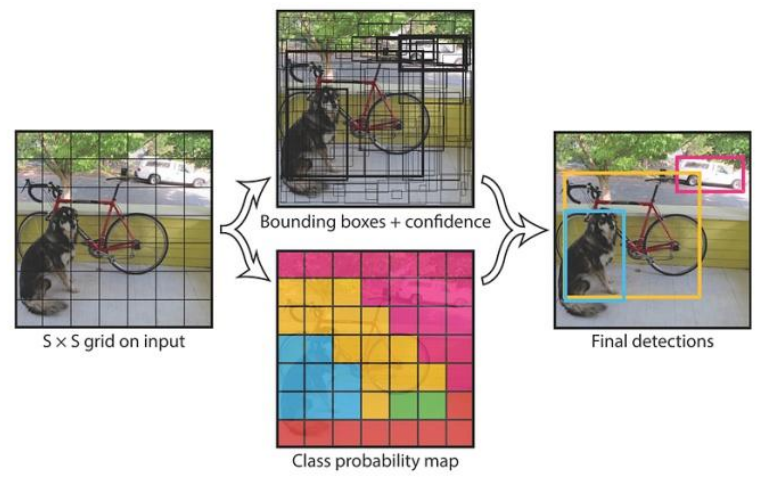

Figure-2: Image detection principle [10]

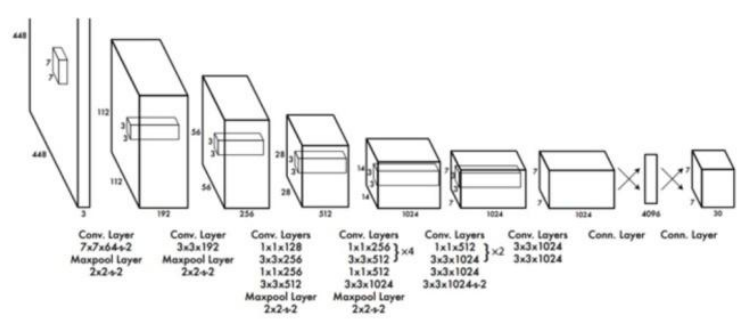

Figure-3: Block diagram of YOLOv3 algorithm [10]

\subsection{Training}

All experiments have been performed on a high performance platform equipped with Ubuntu 16.04 version 30.0 with 4 cores, 2.30GHz CPU and a RAM of $61 \mathrm{~GB}$, MXNet-1.6.0, TensorFlow-2.2.0, 2.1.0 \& 1.15.2, PyTorch-1.4.0 \& 1.5.0, EI, Neuron, \& others. NVIDIA CUDA, cuDNN, NCCL, Intel MKL-DNN, Docker, NVIDIA-Docker \& EFA. The model was trained in Supervisely with linked Supervisely cluster with a learning rate of 0.0001 and epochs were set for 5 .

\section{RESULTS AND DISCUSSION}

Researchers have used YOLOv3 for detecting different objects related to their interest. However, this paper is to check how the YOLOv3 neural network behaves in detecting tigers.

10 images were used from the test dataset of the template matching approach for testing the trained YOLOv3 model and the detection results are examined one by one. If the human eye recognizes the tiger it's considered as ground truth. The minimum confidence threshold was set to 0.5 .

Table-1 depicts the result of our findings.

Table-1: Validating the results of YOLOv3 with Template matching approach for Tiger detection.

\begin{tabular}{|c|c|c|c|c|c|}
\hline \multirow[b]{2}{*}{ Image } & \multirow{2}{*}{$\begin{array}{c}\text { Size } \\
\text { (pixel) }\end{array}$} & \multicolumn{3}{|c|}{ Edge Detection Method } & \multirow[b]{2}{*}{ YOLOv3 } \\
\hline & & $\begin{array}{c}\text { Cann } \\
\mathbf{y}\end{array}$ & $\begin{array}{c}\text { Laplacia } \\
\mathbf{n}\end{array}$ & Sobel & \\
\hline 1 & $640 \times 360$ & True & True & True & True \\
\hline 2 & $840 \times 507$ & True & False & True & True \\
\hline 3 & $780 \times 440$ & False & False & False & False \\
\hline 4 & $800 \times 600$ & True & True & True & True \\
\hline 5 & $340 \times 340$ & False & False & False & True \\
\hline 6 & $750 \times 500$ & False & False & True & True \\
\hline
\end{tabular}

\begin{tabular}{|c|c|c|c|c|c|}
\hline 7 & $274 \times 184$ & False & False & False & False \\
\hline 8 & $670 \times 335$ & True & True & False & True \\
\hline 9 & $670 \times 335$ & False & True & True & True \\
\hline 10 & $480 \times 360$ & True & True & False & True \\
\hline \multicolumn{2}{|c|}{ Accuracy } & $50 \%$ & $50 \%$ & $50 \%$ & $\mathbf{8 0 \%}$ \\
\hline
\end{tabular}

The detector has achieved $\mathbf{8 0 \%}$ precision in all 10 images. Figure 4-13 shows the results of YOLOv3 detector.

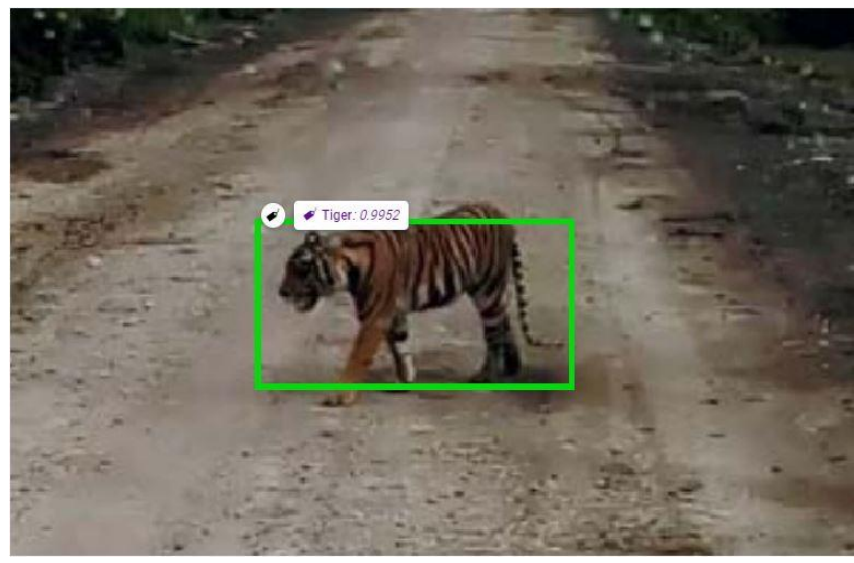

Figure-4: 'True' prediction for image 1 with a confidence score of 0.9952 .

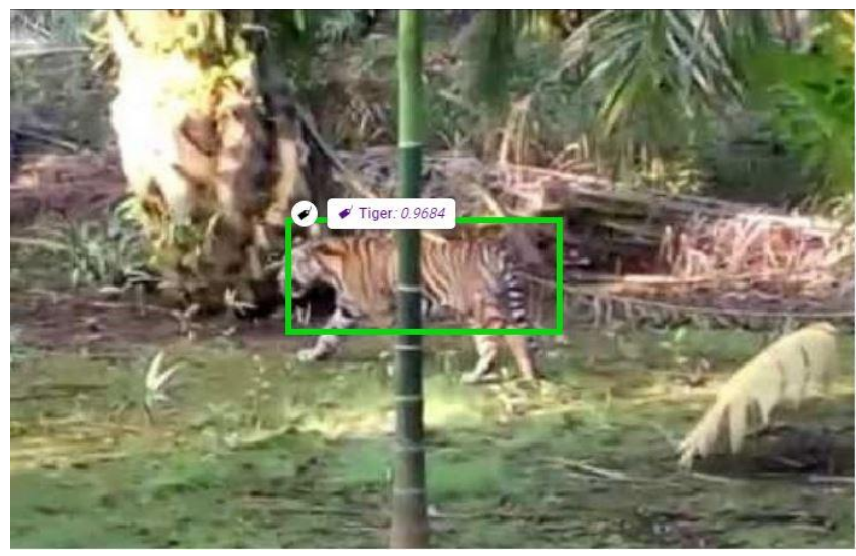

Figure-5: 'True' prediction for image 2 with a confidence score of 0.9684

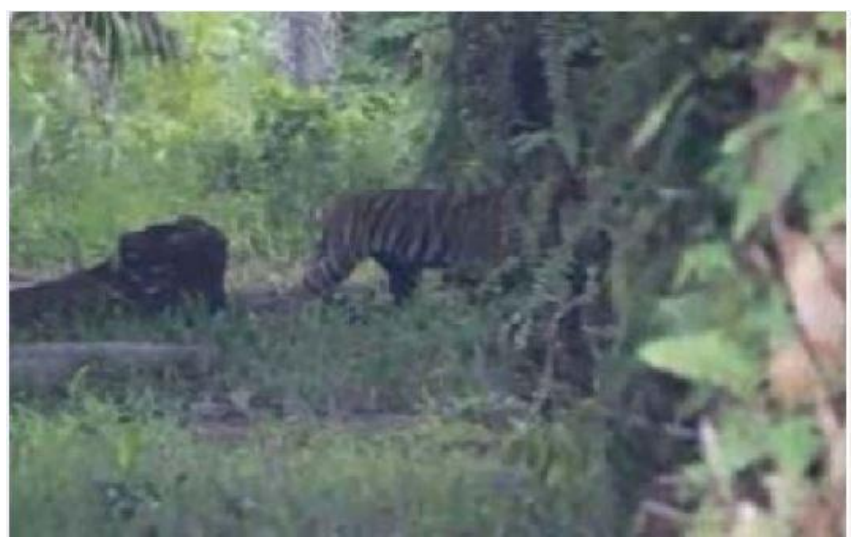

Figure-6: 'False' prediction for image 3 as the minimum confidence threshold was set to 0.5 . 


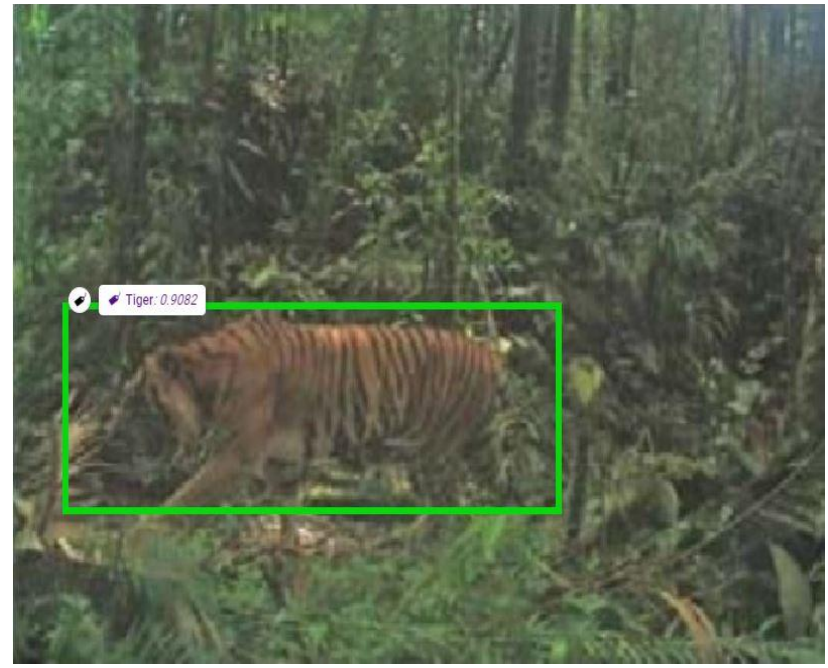

Figure-7: 'True' prediction for image 4 with a confidence score of 0.9082 .

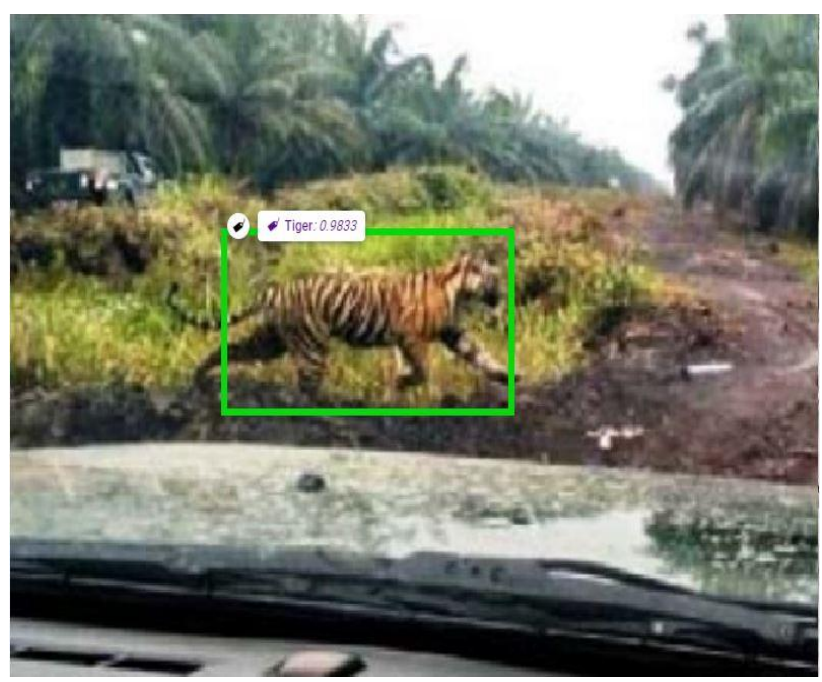

Figure-8: 'True' prediction for image 5 with a confidence score of 0.9833 .

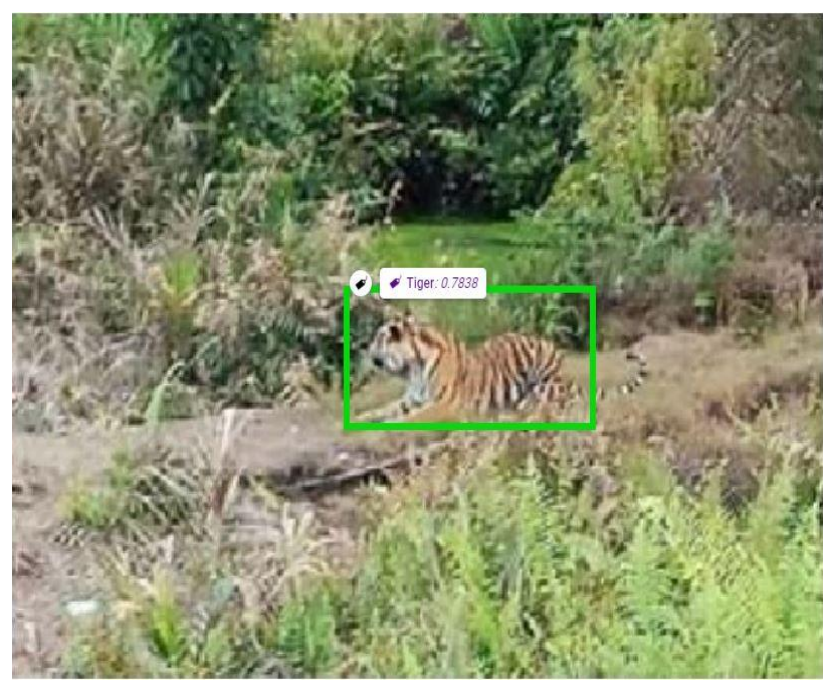

Figure-9: 'True' prediction for image 6 with a confidence score of 0.7838 .

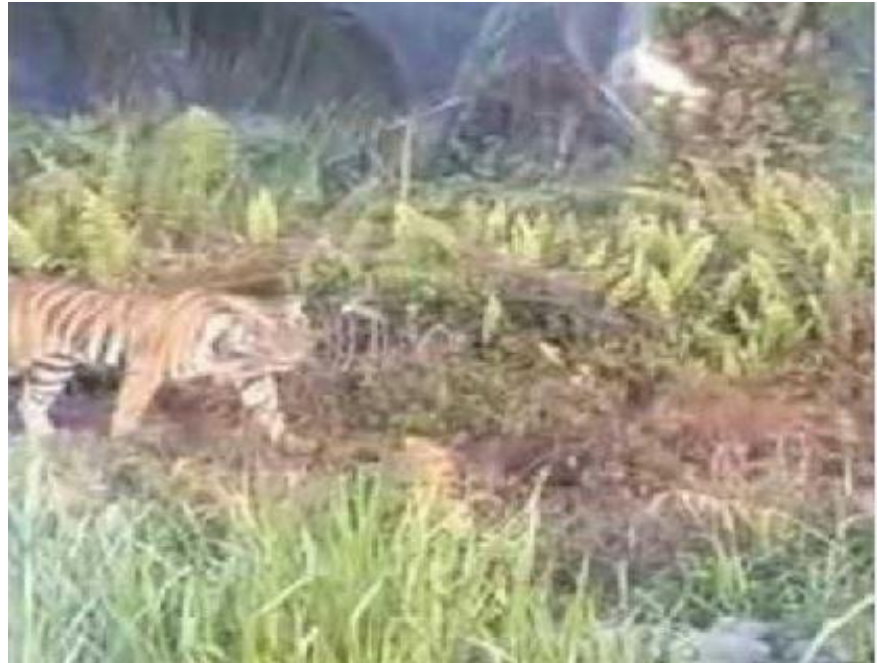

Figure-10: 'False' prediction for image 7 as the minimum confidence threshold was set to 0.5 .

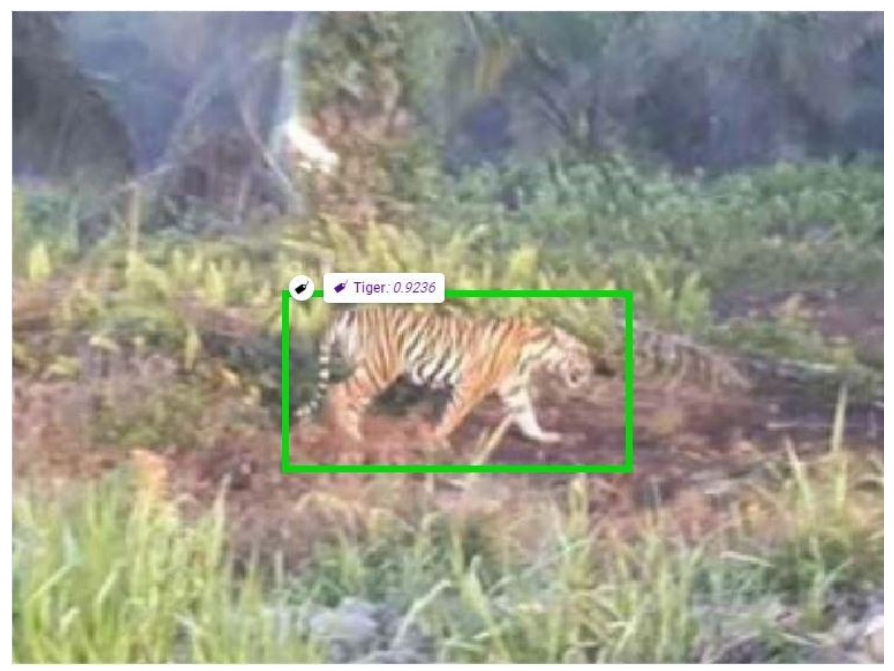

Figure-11: 'True' prediction for image 8 with a confidence score of 0.9236 .

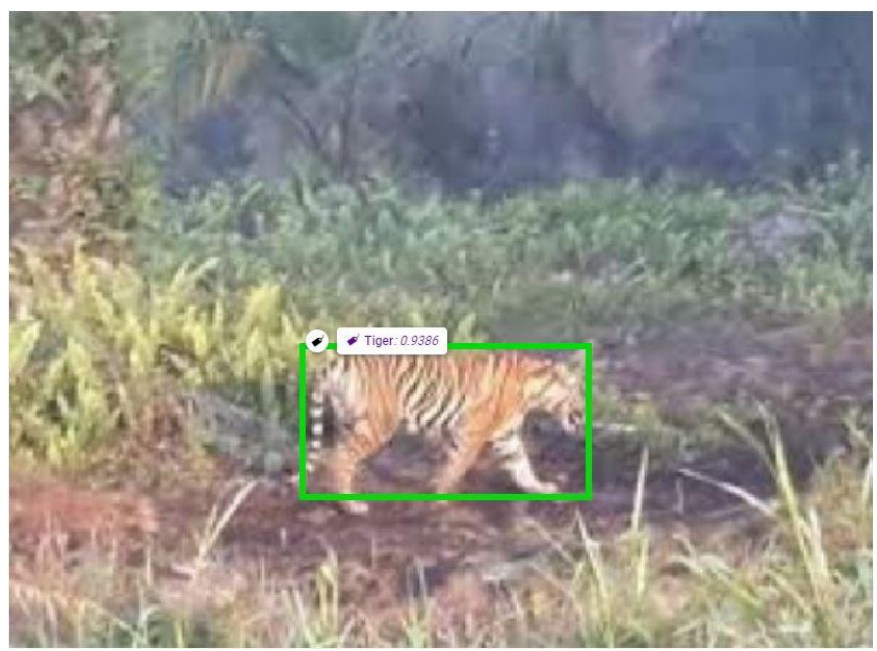

Figure-12: 'True' prediction for image 9 with a confidence score of 0.9386 . 


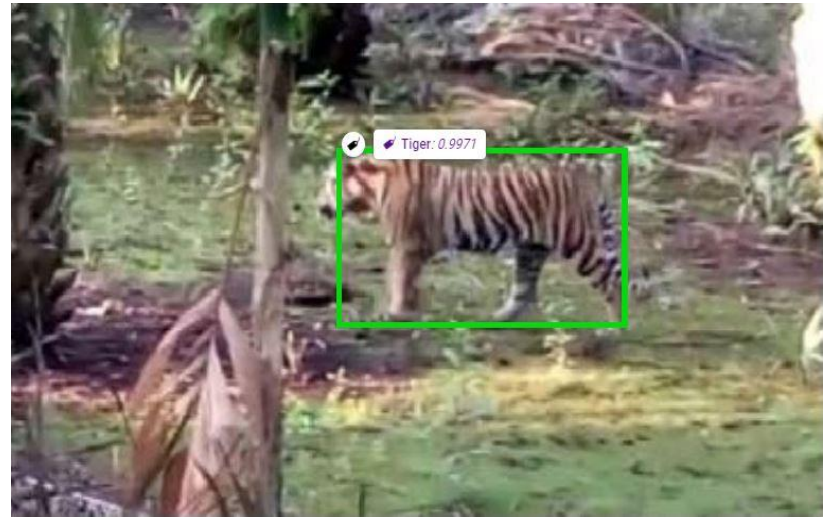

Figure-13: 'True' prediction for image 10 with a confidence score of 0.9971 .

\section{CONCLUSION}

This paper evaluated the performance of YOLOv3 based detector on 10 different images. The objective was to minimize the false positive using YOLOv3 algorithm. It is evident in the result that YOLOv3 gained much more precision than the precision of the template matching approach based on edge patterns. So, use of YOLOv3 is feasible and this model can be applied in a real-time environment after training with a production-ready dataset of large amount. Increased accuracy can be achieved by data augmentation.

\section{REFERENCES}

[1] Wikipedia, The Free Encyclopedia, s.v. "Tiger Hunting," (accessed July 20, 2020), https://en.wikipedia.org/wiki/Tiger_hunting\#Hunting_an d_poaching.

[2] Bdnew24, News Portal , Published: 27 Jan 2018 04:56 AM BdST, (accessed July 20, 2020): (https://bdnews24.com/wildlife/2018/01/27/thirteentigers-killed-in-15-years-in-bangladesh).

[3] Redmon J, Farhadi A. YOLOv3: An Incremental Improvement[J]. arXiv preprint arXiv: 1804.02767, 2018.

[4] Supervisely Online: (https://supervise.ly/).

[5] E Hariyanto et al 2019 J. Phys.: Conf. Ser. 1196012025.

[6] Khan, M. (2009). Can domestic dogs save humans from tigers Panthera tigris? Oryx, 43(1), 44-47. doi:10.1017/S0030605308002068

[7] S. Yanan, Z. Hui, L. Li and Z. Hang, "Rail Surface Defect Detection Method Based on YOLOv3 Deep Learning Networks," 2018 Chinese Automation Congress (CAC), Xi'an, China, 2018, pp. 1563-1568, doi: 10.1109/CAC.2018.8623082.

[8] Y. Yang, X. Huang, L. Cao, L. Chen and K. Huang, "Field Wheat Ears Count Based on YOLOv3," 2019 International Conference on Artificial Intelligence and Advanced Manufacturing (AIAM), Dublin, Ireland, 2019, pp. 444-448, doi: 10.1109/AIAM48774.2019.00094.

[9] A. Warsi, M. Abdullah, M. N. Husen, M. Yahya, S. Khan and N. Jawaid, "Gun Detection System Using Yolov3," 2019 IEEE International Conference on Smart Instrumentation, Measurement and Application (ICSIMA), Kuala Lumpur, Malaysia, 2019, pp. 1-4, doi: 10.1109/ICSIMA47653.2019.9057329.

[10] Introduction to the Principle of YOLO Target Detection Model, Blog , Published: 02 Feb 2019, (accessed July 20, 2020): (https://developpaper.com/introduction-to-theprinciple-of-yolo-target-detection-model/). 